\title{
Performance Analysis of RN-LEACH Protocol over LEACH Protocol
}

\author{
Piyush Rawat* and Siddhartha Chauhan \\ Department of Computer Science and Engineering \\ National Institute of Technology, Hamirpur \\ H.P., India \\ *psh.rawat@gmail.com,sid@nith.ac.in
}

\begin{abstract}
The network lifetime is considered as the major concern in the area of WSN because of the huge quantity of sensor nodes and their distribution in the network. When the size of the network gets expanded the selection of the routing protocol become a crucial issue. A good routing protocol can help in enhancing the network lifetime. In this article, we are exhibiting a routing protocol which is based on existing LEACH protocol named as Random Number based LEACH (RN-LEACH) protocol. The results generated through simulation illustrate that the RN-LEACH algorithm has enhanced the working of the network in terms of energy consumption, network lifetime and packet received compared to existing LEACH protocol. The RN-LEACH protocol has increased the network lifetime by approximately more than $90 \%$ as compared to LEACH which shows the good agreement of RN-LEACH algorithm as contrasted to the previous technique.
\end{abstract}

Keywords: Clustering; Network Lifetime; Energy Efficiency; Routing protocol; Cluster based Protocol

\section{Introduction}

The Wireless Sensor Network (WSN) is a vast accumulation of tiny battery-powered sensor nodes which are furnished with sensing capabilities. The communication between the nodes is performed over wireless [1]. Nowadays wireless sensor network is being used in various areas and applications such as military tracking, environment monitoring, health monitoring etc. The nodes in the wireless network are self-manageable means after deployment these nodes can organize themselves by their own into a network[2]. After the deployment, the sensor nodes can work without human involvement in the network as the deployment of these nodes in a remote area is a very difficult task. To increase the performance of network various techniques are being used so that the energy usage can be minimized and network lifetime can be increased [3]. Clustering is one of the techniques which is used to enhance the performance of the network. In clustering, the nodes are grouped in form of various clusters. In each cluster, a cluster head $(\mathrm{CH})$ is assigned which does the job of gathering data from the members of the cluster. Every node that belongs to the cluster transfer the data to its nearest cluster head. The cluster head after collecting the data performs data aggregation and then transfer that collected information to the base station(BS). It reduces the number of packet transfer to the BS and also minimizes the energy usage of the network. Figure 1 shows the data transfer from various cluster head to the sink.

The routing techniques based on clustering are energy efficient. In Clustering protocols, the nodes with higher energy levels are randomly selected for gathering and transferring

Received (March 10, 2018), Review Result (May 14, 2018), Accepted (May 17, 2018)

* Corresponding Author 
data to $\mathrm{CH}$ whereas the nodes with low energy levels contribute to sensing process[4]. The cluster oriented routing helps in improving various factors in WSN such as scalability, network lifetime, energy usage etc. As there is no determined infrastructure in WSN, the nodes in the network can crash.

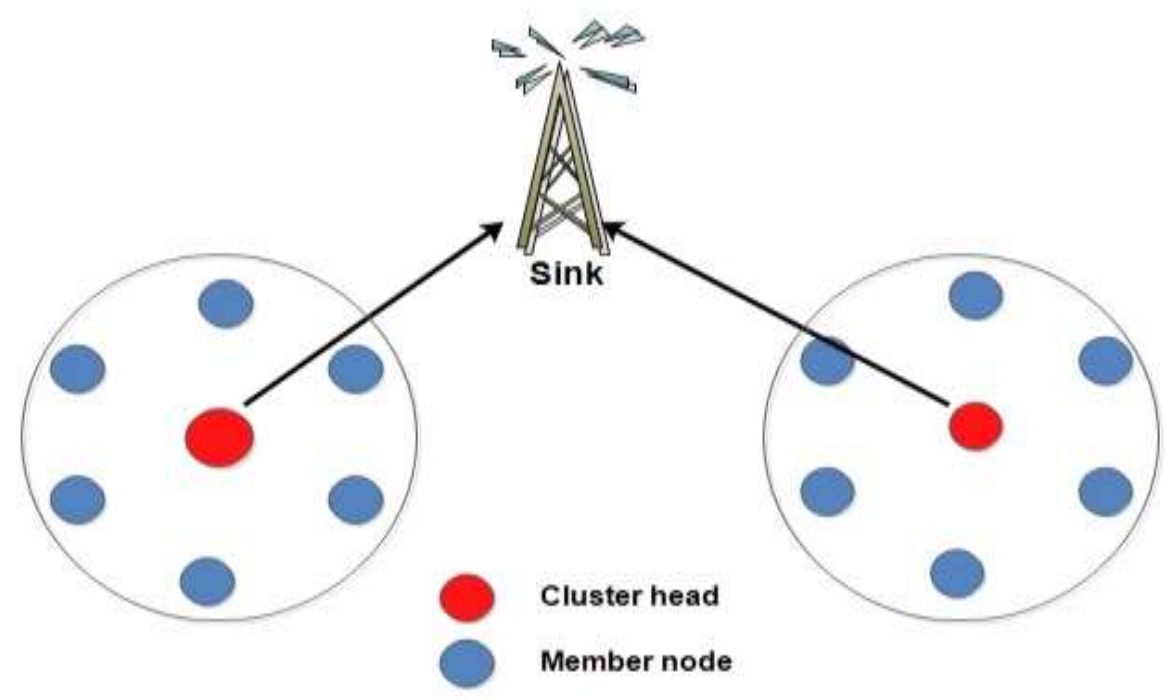

Figure 1. Clustering Process in WSN

So the routing methods have to go through stringent energy-saving provisions[5]. In order to avoid fast node failure, the responsibility of cluster head in clustering protocols are randomly rotated among all the sensor node so that every node can get the chance of becoming cluster head [6].

\section{Related Work}

In [7], [8] a routing protocol is proposed known as Low-Energy Adaptive Hierarchy Protocol(LEACH) which is grounded on homogeneous kind of network where each node in the network have the similar level of energy. In [9], [10] the random selection of node is performed to select $\mathrm{CH}$ and divide the energy among them in a uniform manner. In [11], [12] a variant of LEACH is proposed where the protocol is examined based on several factors such as network life, energy expenditure, and throughput. In [13], [14] the cluster head selection of LEACH is performed in a randomized way. In [15] author proposed a variation of LEACH in which the node with the highest energy is elected to forward data to the BS. In [16] LEACH is modified using MIMO based technique in which cluster head can receive data from various nodes. In [17] the proposed version of LEACH protocol mainly concentrates on throughput and energy of the nodes. In this paper we are proposing a modified version of LEACH protocol called as RN-LEACH which improves the performance factors in WSN like energy consumption, network lifetime, number of packets received, average no. of packets received compared to previous versions.

\section{Performance Factors in WSN}

To measure the performance of WSN various factors like the number of nodes, network topology, and residual energy are used. These factors further help to enhance the performance measures like network lifetime, energy usage and throughput of the network. These factors play a significant role in defining the efficiency of the network. Figure 2 shows some of the key factors of performance in WSN. 


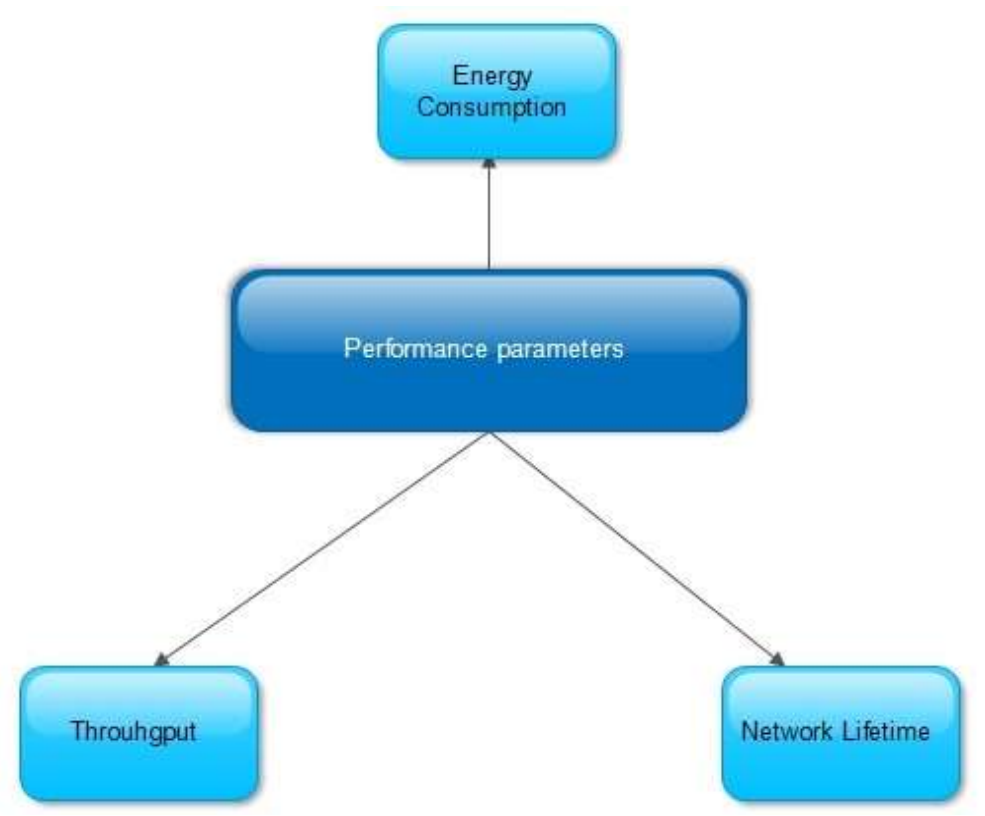

Figure 2. Performance Parameters of WSN

\subsection{Throughput}

In general, throughput is dealing with data that a scheme can handle in a time interval. The throughput of the network is basically defined as the aggregate data packets transferred to the destination per second.

\subsection{Energy Consumption}

It is described as the aggregate energy spent by all the nodes in the network. The energy model has two sections one is for receiving and another one is for transmission. Both the transmitter and receiver are separated by a distanced [18]. Figure 3 shows the energy model used in WSN.

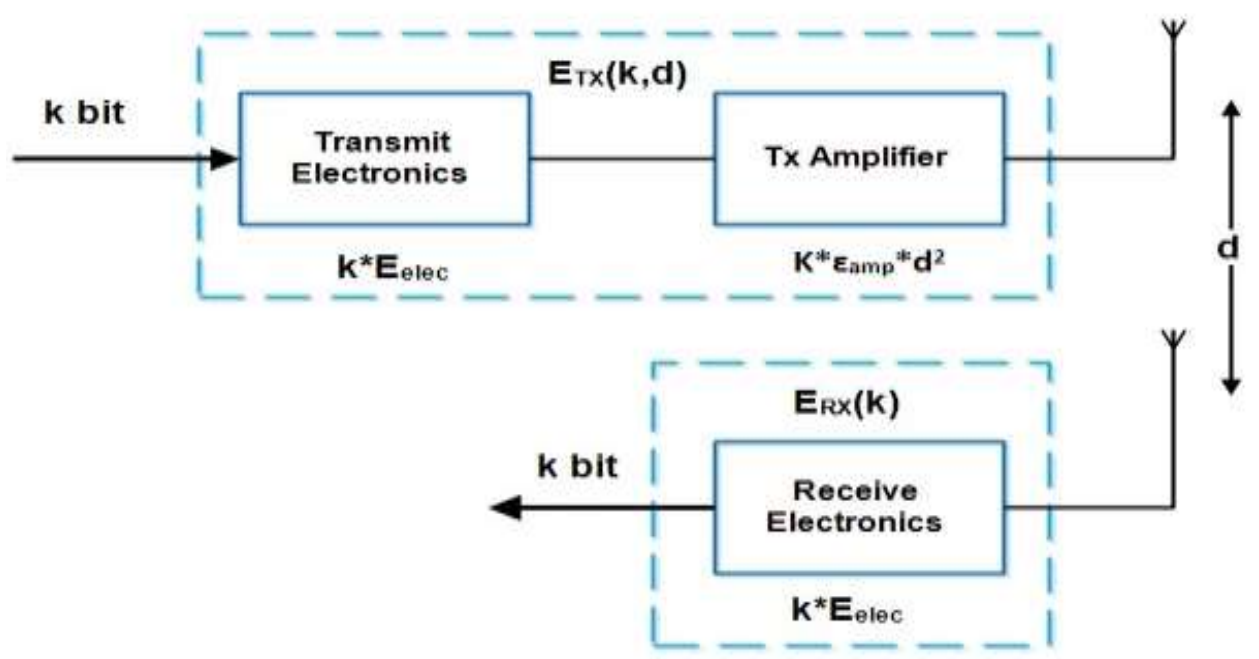

Figure 3. Energy Model of WSN

The energy spends in the transmit and receive of $\mathrm{k}$ bits of data by the sensor nodes is given by the formulas in equation (1) and (2). 
$E_{t x}(k, d)=\left\{\begin{array}{c}k E_{\text {elec }}+k \varepsilon_{\text {efs }} d^{2}, d<d_{o} \\ k E_{\text {elec }}+k \varepsilon_{\text {amp }} d^{4}, d \geq d_{o}\end{array}\right.$

$E_{r x}(k)=k E_{\text {elec }}$

Where,

- $\mathrm{E}_{\mathrm{tx}}$ is the transmission energy and $\mathrm{E}_{\mathrm{rx}}$ is the energy spend in receiving data

- $E_{\text {elec }}$ is the amount of energy required for sending a bit of data. It is used in filtering and modulation

- $\varepsilon_{a m p}$ and $\varepsilon_{e f s}$ are the coefficient of amplification or amplification energy

- $\mathrm{k}$ represents the total data bits and d denotes the separation between receiver and transmitter

\subsection{Network Lifetime}

It is described as the time epoch from the inception of the network till the time when the network becomes nonfunctional [19]. In [20] the author has explained some points related to the network lifetime which are as follows:

- The round when the first sensor node die is denoted as first node dead(FND)

- The round when the number of sensor nodes dead is half of the total nodes is denoted as half node dead(HND)

- The round when last of the sensor nodes expired is denoted as half node dead(LND)

\subsection{Success Rate}

It is described as the ratio of the number of packets acknowledged at the base station to the number of packets transferred from the nodes.

\section{Versions of LEACH}

\subsection{LEACH}

One of the significant outline objectives of WSN is to perform data communication with the aim of increasing the lifetime of the network but without degrading the network performance by applying various energy control procedures [1]. The lifetime of the network has always been a significant concern during the study of WSN. The life of network is exclusively reliant on the battery life of sensors and to enhance the lifetime various algorithms comes into the picture that has been a foremost concern in WSN.

The clustering process is basically divided into two categories based on the energy of sensors nodes as homogeneous clustering and heterogeneous clustering. In the heterogeneous type of clustering, the nodes in the network have different energy levels. While in case of homogeneous clustering all the nodes in the network have same energy levels. In LEACH protocol the process of clustering is divided into phases. Firstly it goes through the set-up phases then it is followed by the steady phase[21].

4.1.1. Setup Phase: The main task of this stage is to form clusters and randomly select the $\mathrm{CH}$ for each of the formed clusters. The selection process of the cluster head is decided on the basis of the generated random value by the nodes that lie between of 0 and 1 and then comparing that random value with the value of threshold function $T(n)$. If the value of random number is lesser than threshold the node becomes $\mathrm{CH}$. In the setup phase, the $\mathrm{CH}$ advertises a message to every node in its range to inform that it has become $\mathrm{CH}$. The threshold function is given as: 


$$
\mathrm{T}(n)=\left\{\begin{aligned}
\frac{P}{1-P\left[\left(\operatorname{rmod} \frac{1}{P}\right)\right]}, & \text { if } n \in G \\
0, & \text { otherwise }
\end{aligned}\right.
$$

Where

- $r$ represents the current round

- $\mathrm{P}$ represents the probability of selecting $\mathrm{CH}$

- $\mathrm{G}$ represents the set of nodes who do not get chance of becoming $\mathrm{CH}$ from last $1 / \mathrm{p}$ rounds

4.1.2. Steady Phase: During this phase, the cluster member nodes transfer their sensed information to the $\mathrm{CH}$ which is situated at one hop distance from the nodes. Each node sends data in their respective time slots. The $\mathrm{CH}$ collects data from every node in the cluster and performs aggregation of data. The data produced after the aggregation process is then transferred to the base station by the $\mathrm{CH}$.

\subsection{RN-LEACH}

In the proposed technique the main focus is on increasing lifetime of network and minimizing the consumption of energy in the network. The foremost advantage of RNLEACH is that it augments the lifespan of system, increases packets conveyed to BS and enriches the period of stability in network contrasted to other existing techniques. In LEACH protocol during the process of cluster head election, a random number is generated by the nodes which lie between 0 and 1 and then matches that random value with the value of threshold function. But in that procedure, there is no involvement of energy of the nodes. In RN-LEACH we will modify the process of random number generation by the nodes. The generated random number is multiplied by the ratio of remaining energy to the initial energy of the nodes. Figure 4 shows the steps used in the RN-LEACH protocol. It is representing the flowchart of the proposed model.

In RN-LEACH to increase the network lifetime, random number is modified and the comparison is done with threshold function for cluster head selection. The random number generated is denoted as rand(n). The modified random number in RN-LEACH is given as:

$\operatorname{rand}(n)^{\prime}=\operatorname{rand}(n) * \frac{\text { remaining energy }}{\text { initial energy }}$

The modified random number can be also represented as:

$\operatorname{rand}(n)^{\prime}=\operatorname{rand}(n) * \frac{E O-S(i) \cdot E}{E o}$

Where,

- $\mathrm{n}$ is the node

- Eo is the primary energy assigned to each node

- $S(i) . E$ represents the current energy of node 


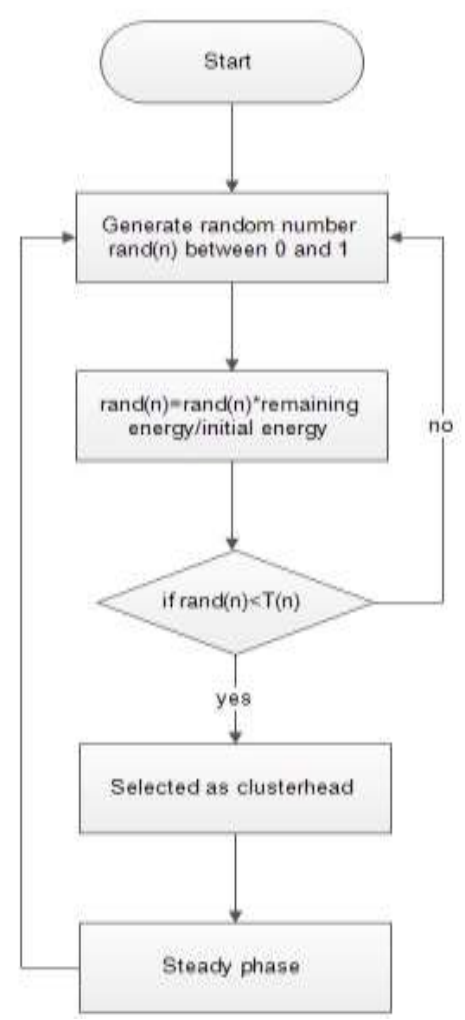

Figure 4. Flowchart of RN-LEACH

\section{Simulations and Results}

In this article, we are using the MATLAB simulator to measure the execution of the system based on a few parameters. The parameters and variables utilized during the simulation are shown in Table 1 and Table 2. The simulation results show the comparison between the LEACH and RN-LEACH based on different performance factors.

Table 1. Simulation Factors and their Values

\begin{tabular}{|l|l|}
\hline Parameters & Value \\
\hline EDA & $5 \mathrm{~nJ} / \mathrm{bit}$ \\
\hline$\varepsilon_{\mathrm{efs}}$ & $8 \mathrm{pJ} / \mathrm{bit} / \mathrm{m} 2$ \\
\hline$\varepsilon_{\mathrm{amp}}$ & $0.0012 \mathrm{pJ} / \mathrm{bit} / \mathrm{m} 4$ \\
\hline Eelec & $40 \mathrm{~nJ} / \mathrm{bit}$ \\
\hline $\mathrm{k}$ & 4000 \\
\hline
\end{tabular}

\section{Table 2. Simulation Variables and their Initial Values}

\begin{tabular}{|l|l|}
\hline Simulation Variables & Initial Values \\
\hline Size of network & $100 \mathrm{~m}^{*} 100 \mathrm{~m}$ \\
\hline Initial Energy & $0.5 \mathrm{~J}$ \\
\hline Total nodes & 100 \\
\hline Rounds & 4000 \\
\hline
\end{tabular}

Some of the assumptions are taken into account while carrying out the simulation which is as follows:

- Every node in the network have the same amount of energy means the network is of homogeneous type 
- The nodes in the network are not mobile in nature

- The data packets must be communicated properly by the sensor nodes and they are received by the base station without any failure

- Only around $10 \%$ of the nodes in the network can become $\mathrm{CH}$ in each round because the initial value of $\mathrm{p}$ is set to 0.1

The performance observation of LEACH and RN-LEACH is performed on the basis of various factors such as network lifetime, energy consumption and packets received. On the basis of these factors, we can judge the performance of RN-LEACH in comparison to LEACH protocol.

In Figure 5 the comparison is performed between the RN-LEACH and LEACH on the basis of their network lifetime. The graph is plotted between the number of alive nodes vs the round number. On the basis of the graph, we can observe that the nodes in the LEACH sustained till 1853 rounds only but in RN-LEACH the last node died in 3622 rounds. From the figure, it can be clearly portrayed that the RN-LEACH has enhanced the network lifetime as compared to LEACH.

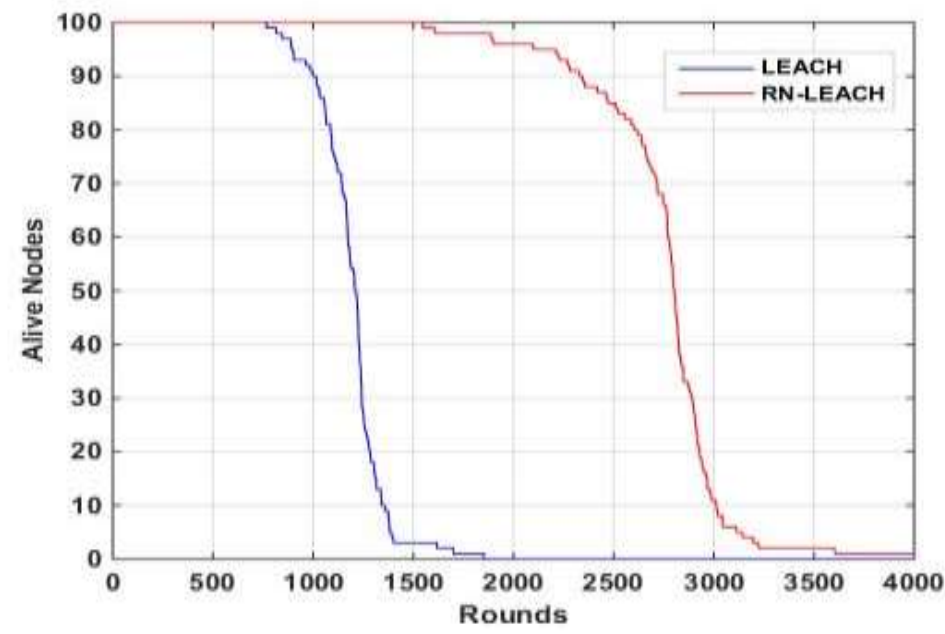

Figure 5. Lifetime of Network

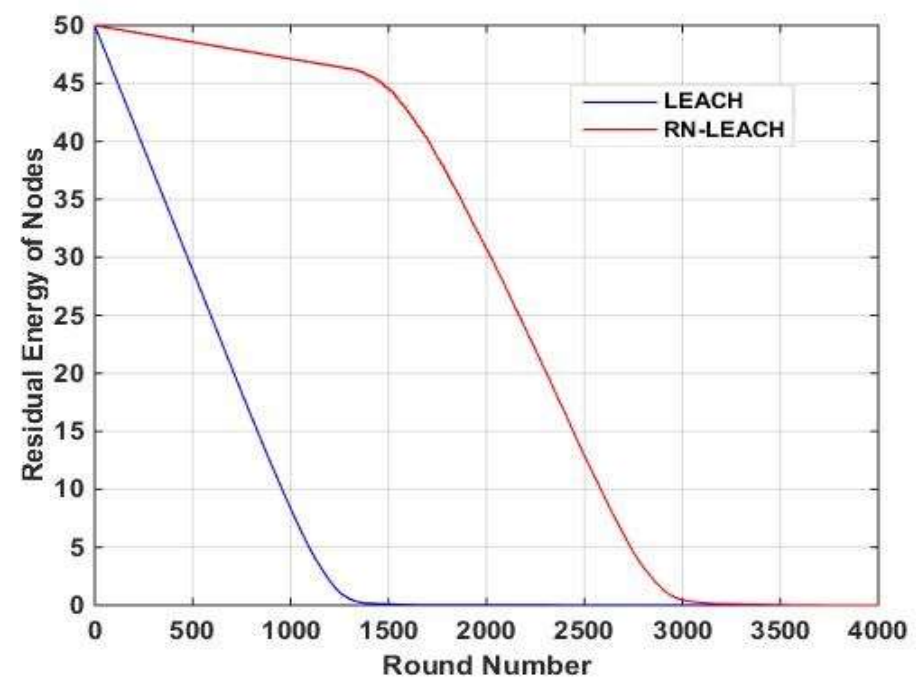

Figure 6. Residual Energy of Nodes 
Figure 6 represents the residual energy of RN-LEACH and LEACH protocol. The graph is plotted between the remaining energy and the round number. The figure shows how much energy is consumed in each round. The initial energy of the network is taken as 50J that keeps on degrading in each round. The energy level in LEACH protocol ends up in approximately 1437 round while in RN-LEACH protocol it ends up in approximately 3170 rounds. Hence it shows that the performance of RN-LEACH is better than existing LEACH.

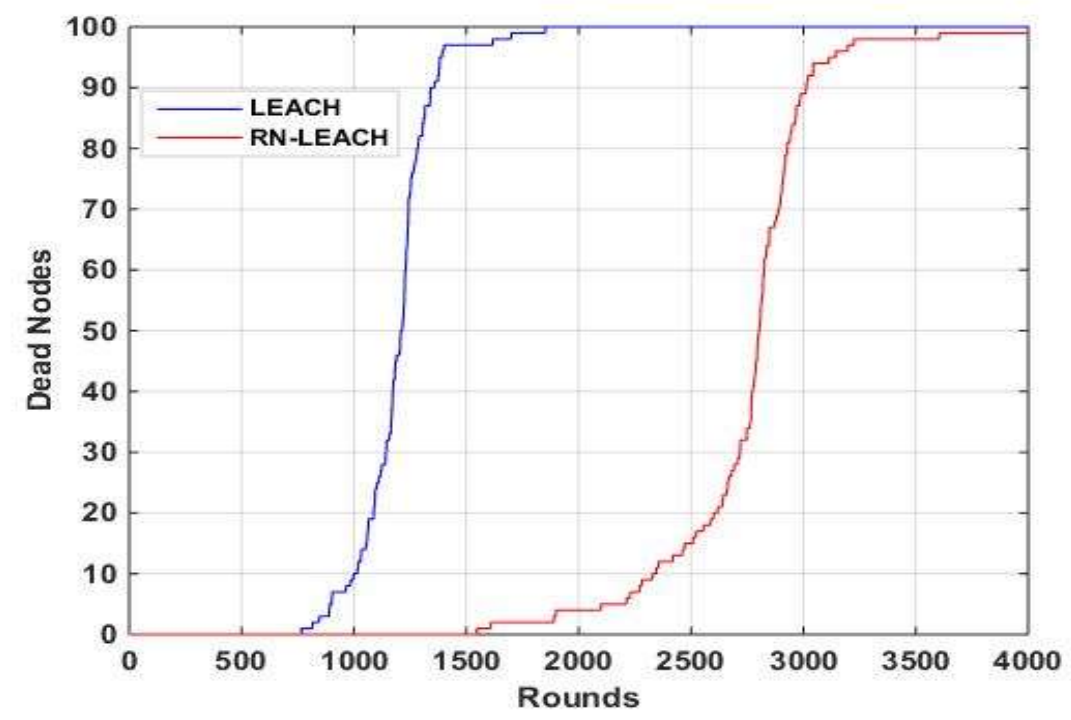

Figure 7. Dead Nodes in Each Round

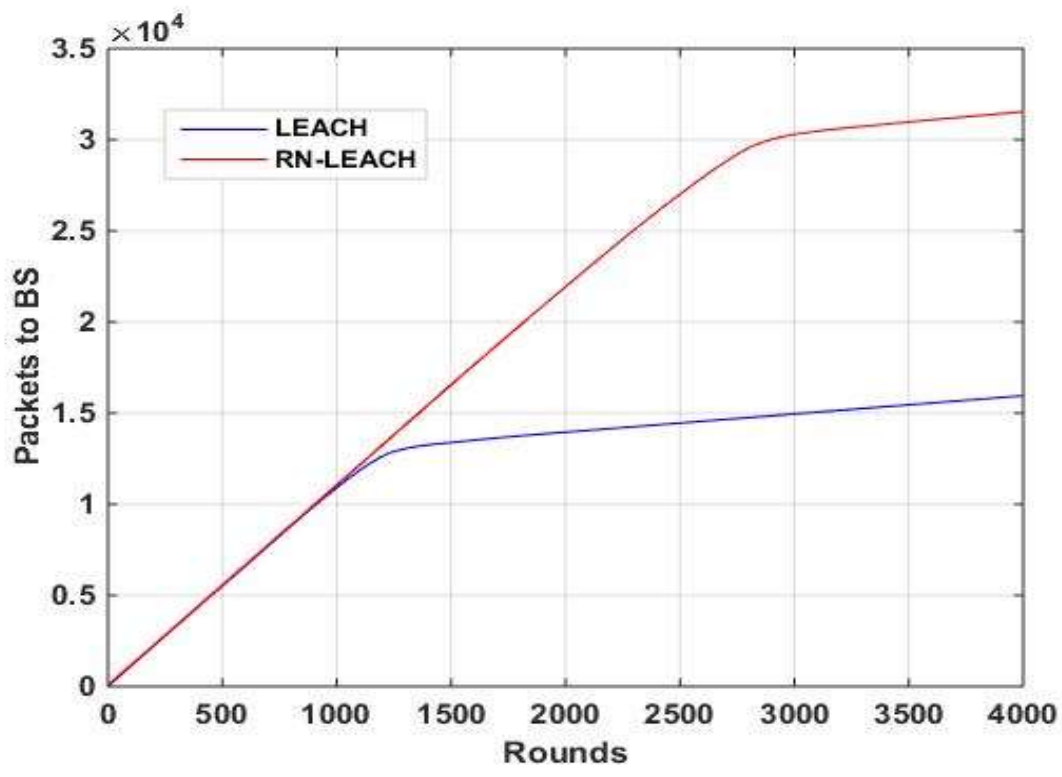

Figure 8. Packets to Base Station

Figure 7 represents the dead nodes in the network. The graph is plotted between the number of dead nodes vs the number of rounds. It can be portrayed from the graph that the lifetime of nodes in the RN-LEACH is more as compared to the LEACH.

Figure 8 represents the received packets by the BS in RN-LEACH and LEACH protocol. The graph shown is plotted between packets received by the base station versus the round number. In other words, we can say that Figure 8 is representing the throughput of the 
network in both the protocols. It can be clearly depicted that the total packets acknowledged by BS in RN-LEACH are higher in resemblance to the LEACH protocol. Till 10000 rounds the number of packets is same in both the protocols. But with the increase in the number of rounds the packets received in LEACH becomes constant, on the other hand in case of RNLEACH the number of packets received by the BS keeps on increasing with rounds. So we can conclude that the RN-LEACH protocol has enhanced the network lifetime contrasted to the LEACH protocol.

\section{Conclusion}

In this article, various factors for performance, for example, energy consumption, network lifetime and packets to the base station are used with different parameters in the system. These performance measures of RN-LEACH and LEACH are compared based on these parameters. The results of the simulation illustrate that the network lifetime of RNLEACH is better as compared to LEACH protocol and energy consumption in RN-LEACH is less than LEACH. And also the data packets received in RN-LEACH is greater than LEACH protocol. Furthermore, the RN-LEACH protocol shows a satisfactory performance improvement as contrasted to LEACH protocol. This research proposal can be further extended to examine several other parameters such as latency and packet delivery ratio. It would be interesting to further explore these parameters with different clustering techniques and compare them with other previous works.

\section{References}

[1] J. N. Al-Karaki and A. E. Kamal, "Routing techniques in wireless sensor networks: a survey", IEEE Wirel. Commun., vol. 11, no. 6, (2004) December, pp. 6-28.

[2] D. Goyal and M. R. Tripathy, "Routing Protocols in Wireless Sensor Networks: A Survey", 2012 Second International Conference on Advanced Computing \& Communication Technologies, (2012), pp. 474-480.

[3] J. Yick, B. Mukherjee and D. Ghosal, "Wireless sensor network survey", Comput. Networks, vol. 52, no. 12, (2008) August, pp. 2292-2330.

[4] S. P. Singh and S. C. Sharma, "A Survey on Cluster Based Routing Protocols in Wireless Sensor Networks", Procedia Comput. Sci., vol. 45, (2015) January, pp. 687-695.

[5] K. Akkaya and M. Younis, "A survey on routing protocols for wireless sensor networks", Ad Hoc Networks, vol. 3, no. 3, (2005) May, pp. 325-349.

[6] X. Liu and Xuxun, "A Survey on Clustering Routing Protocols in Wireless Sensor Networks", Sensors, vol. 12, no. 8, (2012) August, pp. 11113-11153.

[7] W. B. Heinzelman, A. P. Chandrakasan and H. Balakrishnan, "An application-specific protocol architecture for wireless microsensor networks", IEEE Trans. Wirel. Commun., vol. 1, no. 4, pp. 660-670, (2002) October.

[8] E. Hossain and K. K. Leung, "Wireless mesh networks: architectures and protocols", Springer, (2011).

[9] M. Kohvakka, J. Suhonen, M. Kuorilehto, V. Kaseva, M. Hännikäinen and T. D. Hämäläinen, "Energyefficient neighbor discovery protocol for mobile wireless sensor networks", Ad Hoc Networks, vol. 7, no. 1, (2009) January, pp. 24-41.

[10] N. Duy Tan and N. Dinh Viet, "SSTBC: Sleep scheduled and tree-based clustering routing protocol for energy-efficient in wireless sensor networks", The 2015 IEEE RIVF International Conference on Computing \& Communication Technologies - Research, Innovation, and Vision for Future (RIVF), (2015), pp. $180-185$.

[11] T. M. Rahayu, S.-G. Lee and H.-J. Lee, "Survey on LEACH-based security protocols", 16th International Conference on Advanced Communication Technology, (2014), pp. 304-309.

[12] S. K. Singh, P. Kumar and J. P. Singh, “A Survey on Successors of LEACH Protocol”, IEEE Access, vol. 5, (2017), pp. 4298-4328.

[13] D. Zhixiang and Q. Bensheng, "Three-layered routing protocol for WSN based on LEACH algorithm", IET Conference on Wireless, Mobile and Sensor Networks 2007 (CCWMSN07), vol. 2007, (2007), pp. $72-75$.

[14] S. Tyagi and N. Kumar, "A systematic review on clustering and routing techniques based upon LEACH protocol for wireless sensor networks", J. Netw. Comput. Appl., vol. 36, no. 2, (2013) March, pp. 623645.

[15] G. S. Arumugam and T. Ponnuchamy, "EE-LEACH: development of energy-efficient LEACH Protocol for data gathering in WSN", EURASIP J. Wirel. Commun. Netw., vol. 2015, no. 1, (2015) December, pp. 76. 
[16] Asaduzzaman and H. Y. Kong, "Energy efficient cooperative LEACH protocol for wireless sensor networks", J. Commun. Networks, vol. 12, no. 4, (2010) August, pp. 358-365.

[17] R. Priyadarshi, H. Tripathi, A. Bhardwaj, A. Thakur and A. Thakur, "Performance metric analysis of modified LEACH routing protocol in wireless sensor network”, Int. J. Eng. Technol., vol. 7, no. 1-5, (2017) December, pp. 196.

[18] S. Sasirekha and S. Swamynathan, "Cluster-Chain Mobile Agent Routing Algorithm for Efficient Data Aggregation in Wireless Sensor Network", vol. 19, no. 4, (2017), pp. 392-401.

[19] Y. Chen and Q. Zhao, "On the lifetime of wireless sensor networks", IEEE Commun. Lett., vol. 9, no. 11, (2005) November, pp. 976-978.

[20] I. Dietrich, F. Dressler, I. Dietrich and F. Dressler, "On the Lifetime of Wireless Sensor Networks", ACM Trans. Sen. Netw. ACM Trans. Sens. Networks, vol. 5, no. 1, (2009), pp. 39-1.

[21] A. S. Toor and A. K. Jain, "A survey of routing protocols in Wireless Sensor Networks: Hierarchical routing", 2016 International Conference on Recent Advances and Innovations in Engineering (ICRAIE), (2016), pp. 1-6. 